\title{
After a decade of annual dose of mass ivermectin treatment in Cameroon and Uganda, onchocerciasis transmission continues
}

\author{
Moses Katabarwa', Albert Eyamba ${ }^{2}$, Peace Habomugisha ${ }^{3}$, Tom Lakwo ${ }^{4}$, Same Ekobo ${ }^{5}$, Joseph Kamgno ${ }^{6}$, \\ Thomas Kuete $^{5}$, Richard Ndyomugyenyi ${ }^{4}$, Ambrose Onapa ${ }^{4}$, Mkpouwoueiko Salifou ${ }^{7}$, Marcelline Ntep ${ }^{7}$ and \\ Frank O. Richards'
}

1 The Carter Center, Atlanta, GA, USA

2 The Carter Center, Yaoundé, Cameroon

3 The Carter Center, Kampala, Uganda

4 Ministry of Health, Kampala, Uganda

5 Department of Parasitology, School of Medicine, Yaoundé University, Yaoundé, Cameroon

6 Filariasis Research Centre, Yaoundé

7 Ministry of Health, Yaoundé, Cameroon

Summary

овJестіve To evaluate the effectiveness of 10 years' annual single dose ivermectin treatment on onchocerciasis transmission in hyperendemic areas of Cameroon and Uganda.

METHODS Baseline nodule and microfilaria ('skin snip') prevalence data were available from 10 hyperendemic sentinel communities in Cameroon (from 1996) and hyperendemic 20 sentinel communities in Uganda (from 1993). We returned to these villages in 2005, 10 months after the last annual ivermectin distribution, to repeat the cross-sectional surveys. Each sentinel community reported a mean interval treatment coverage of eligible persons of $>88 \%$ (range 37-100\%). Data were analyzed for more than 6200 person examinations. In Cameroon, 719 people $\geq 10$ years were examined at the baseline survey in 1996 and 838 at the follow-up survey in 2005. In Uganda, 1590 people $\geq 10$ years were examined at the baseline survey in 1993 and 2122 people at the follow-up survey in 2005. We also examined children under 10 in Cameroon $(1996, n=185 ; 2005, n=448)$ and Uganda $(1993, n=177 ; 2005, n=130)$. In Uganda, the vitality of worms was judged using standard histological criteria in 80 nodules excised in 2005 .

RESULTS The prevalence of microfilaria carriers among older children and adults ( $\geq 10$ years) in Cameroon sentinel communities dropped from $70.1 \%$ to $7.04 \%(P<0.0001)$ over the 10 -year treatment period; that of nodule carriers from $58 \%$ to $9.55 \%(P<0.0001)$. Similarly, in Uganda, the prevalence of microfilaria carriers fell from $71.9 \%$ to $7.49 \%(P<0.0001)$ over the 13 -year treatment period, and that of nodule carriers from $53.21 \%$ to $9.66 \%(P<0.0001)$. The number of microfilaria carriers among children $<10$ years in Cameroon decreased from $29.73 \%$ to $3.8 \%(P<0.0001)$, and in Uganda from $33.89 \%$ to $3.1 \%(P<0.0001)$. In 2005 , worms excised from nodules in Uganda, $81.4 \%$ of males remained alive, and $64 \%$ of females, with $24 \%$ of them inseminated.

CONCLUSION A decade or more of annual single dose ivermectin treatment in hyperendemic areas has reduced onchocerciasis to 'hypoendemicity', but onchocerciasis transmission persists. For now, annual treatment with ivermectin should be continued in formerly mesoendemic and hyperendemic zones.

keywords onchocerciasis, ivermectin, single annual dose, transmission, prevalence

\section{Introduction}

Onchocerciasis is a leading infectious cause of blindness in Africa and a cause of severe skin disease. It is caused by Onchocerca volvulus, a parasitic worm that is encapsulated in nodules under the skin. Onchocerca volvulus female worms produce microfilariae which exit the nodules, swarm in the dermis and enter the eye causing cutaneous and ophthalmologic complications. Microfilaria picked up by certain Simulium species blackflies during a blood meal develop into infectious stages and are transmitted on subsequent bites. The flies breed in fast-flowing rivers and streams.

Ivermectin is a safe and effective microfilaricidal drug that has been donated by Merck \& Co. since 1987 for administration in mass treatment programmes to control 
M. Katabarwa et al. Onchocerciasis transmission in Cameroon and Uganda

onchocerciasis. When used on an individual basis, ivermectin rapidly kills the microfilariae and reduces the fecundity of adult female worms, but does not kill them (Taylor et al. 1990; Rodriguez-Perez \& Reyes-Villanueva 1994; Chippaux et al. 1995). Therefore, ivermectin must be given repetitively. The frequency and duration of ivermectin administration remains at issue, and is influenced by whether ivermectin mass treatment in a given area can stop new infections (transmission) from taking place.

The Ministry of Health $(\mathrm{MOH})$ onchocerciasis control programmes in Cameroon and Uganda had been assisted by the Carter Center and Lions Clubs since 1996, in partnership with the African Programme for Onchocerciasis Control (APOC) and the affected communities. The goal of the APOC partnership is 'to eliminate onchocerciasis as a disease of public health and socio-economic importance throughout Africa' (Amazigo \& Boatin 2006). The strategy is to deliver an annual dose of ivermectin to the entire eligible population of onchocerciasis meso- and hyperendemic villages through community-directed treatment with ivermectin (CDTI) (Molyneux \& Davies 1997). Mesoendemicity is defined as onchocercal nodule rates $\geq 20 \%$ or microfilardermia rate $>40 \%$; byperendemicity as nodule rates $\geq 40 \%$ or microfilardermia $\geq 60 \%$ (WHO 1991). CDTI is an approach where community members are educated about onchocerciasis and then allowed to organize and rely upon themselves to provide annual treatment (Katabarwa et al. 2002). Community members called community-directed distributors (CDDs) are selected by the community at large and trained by health workers to carry out periodic household census, health educate and treat their fellow community members. CDDs are also trained to manage minor side reactions, and promptly report to the nearest health facility severe reactions, treatment data and drug utilization.

The APOC approach is to provide core financial support from a World Bank Trust Fund to CDTI projects for a period of 5 years to help establish ivermectin delivery through CDTI with the hope that after building the capacity in the project areas, ivermectin distribution will be sustained; some additional APOC support is provided for replacement of capital items and advocacy for 3 years after the 5 -year core period. The duration of treatment required after APOC support ceases to reach the goal of elimination as a public health problem remains an objective of speculation and debate. Some sources suggest that ivermectin distribution should continue for a total of 15 years (Amazigo et al. 2002), based on the estimate that the adult O. volvulus worms live that long. Such a calculation is based on the assumption that transmission of the parasite will be essentially interrupted by annual treatment in mesoand hyperendemic areas. However, studies show that a single annual dose of ivermectin may reduce but not completely stop onchocerciasis transmission, and that recrudescence could occur after 15 years of treatment (Remme et al. 1990; Boatin et al. 1998). Therefore, to prevent recrudescence and maintain the gains made in disease morbidity control, some have argued that ivermectin programmes based on annual doses of ivermectin require indefinite ivermectin distribution (Richards et al. 2000; Winnen et al. 2002). To throw more light on this issue, we assessed the impact of single annual dose of ivermectin on onchocerciasis in 'Post-APOC' areas of Cameroon and Uganda after a decade or more of uninterrupted distribution to assess impact on prevalence and transmission of the parasite. Our fundamental question was, 'Are we reaching a point where it would be safe to halt ivermectin treatment?'

\section{Methods}

Study sites

Pre-treatment (baseline) community level microfilaria and nodule prevalence data were available from 1996 for 10 sentinel communities in West Province, Cameroon, and from 1993 for 20 sentinel communities in several districts in Uganda (Tables 1 and 2). Baseline data for 904 person examinations in Cameroon were obtained from two sentinel communities in each of five health districts: Bangangte, Foumbot, Bafang, Kekem and Bandja. There were no baseline data for children from Bangangte and Bandja districts. In Uganda, baseline data for 1767 person examinations were from five districts: Mbale (four communities), Kasese (three communities), Nebbi (four communities), Kisoro (five communities) and Moyo (four communities). We returned to the same villages in 2005 , 10 months after the previous annual ivermectin distribution, to repeat the cross-sectional surveys using a similar method as in the first surveys. Thus, we evaluated sentinel communities after 10 years of treatment in Cameroon, and 13 years of treatment in Uganda. We examined permanent community members in two groups: (1) 'Older children and Adults' (anyone age 10 years and older), and (2) 'Young Children' (under 10 years of age who had lived their entire lives in the communities and were therefore born into transmission conditions after ivermectin distribution began). Visitors and adults who had not lived in the communities for at least 10 years were excluded from the study.

The programme impact surveys were approved by the $\mathrm{MOH}$ in both countries. Oral consent was obtained after the objectives of the study and the examination process was explained to village chiefs, the community at large and 
M. Katabarwa et al. Onchocerciasis transmission in Cameroon and Uganda

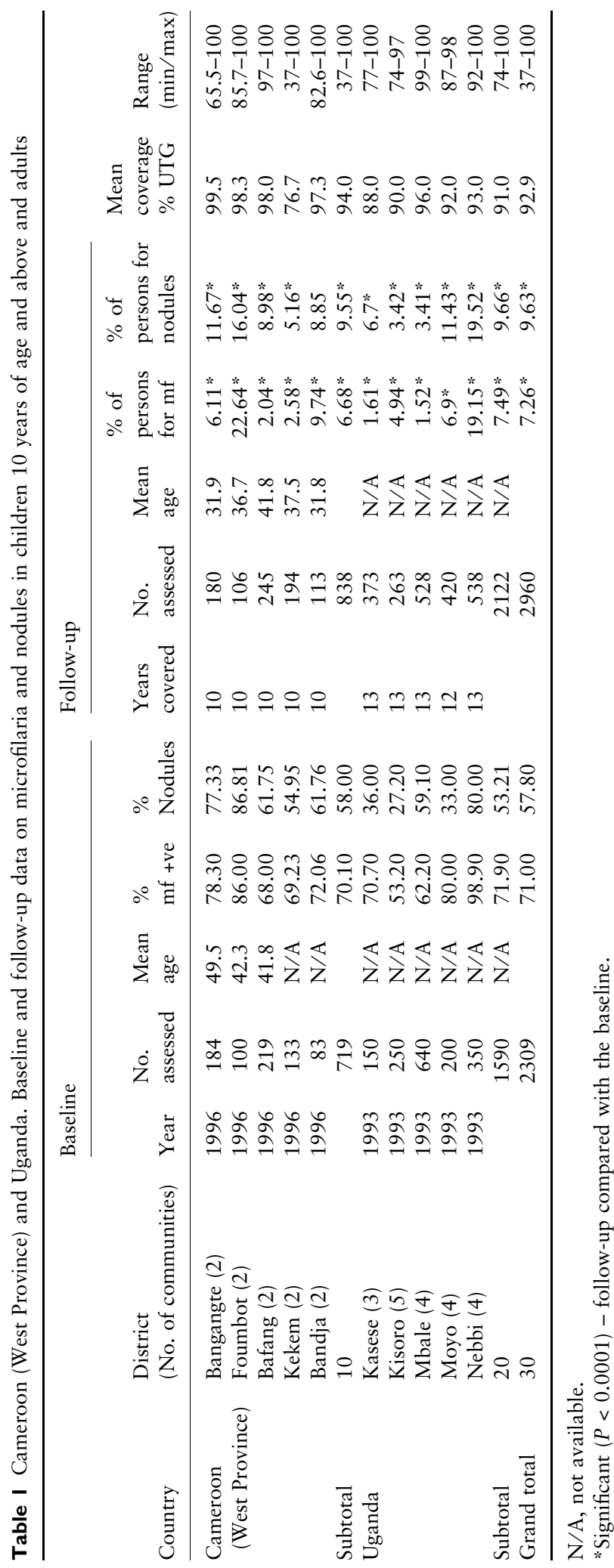

parents of individual children. Trained $\mathrm{MOH}$ personnel carried out the examinations. Participation was voluntary and any individual (or parent of a child) was free to opt out of examination without fear of retaliation from their community leaders and programme personnel.

\section{Qualitative nodule examination}

After name, age (recorded in Cameroon as an age range) and gender were recorded on an individual registration form, each participant was examined in a well-lit private room. Qualified and certified $\mathrm{MOH}$ staff performed a palpation examination on the partially disrobed participant, paying particular attention to bony prominences of the torso, iliac crests and upper trocanter of the femurs. Onchocercal nodules (onchocercomas) were identified clinically as being firm, painless and mobile (Albiez et al. 1988; Ngoumou et al. 1994; Katabarwa et al. 1999). Results were recorded on the form as 'positive' or negative'. Nodule prevalence was expressed as a percent (number positive for nodules divided by number examined $\times 100$ ) and classified as hypoendemic (nodule rate $\leq 20 \%$ ), mesoendemic (nodule rate $\geq 20-40 \%$ ) or byperendemic (nodule rate $\geq 40 \%$ ) (WHO Report 1991).

\section{Qualitative microfilaria examination}

Immediately after the nodule examination, two skin snips were taken from each iliac crest posteriorly (Prost \& Prod'hon 1978) as follows: (1) the site was cleansed with an antiseptic, (2) a 2-3 mg sample of skin was taken with the help of disposable sterile dermal hook and scalpel, (3) the skin sample was placed immediately in wells of microtitration plates containing a sterile normal saline solution, (4) anther snip was taken from the opposite side following the same procedure (1-3), (5) hook and blade were safely discarded, (6) sterile bandages were applied and (7) the wells used were noted on the patient form. When the plate was full, it was sealed with a transparent adhesive tape. After $12-24 \mathrm{~h}$, the snips were removed and the fluid from each well was examined separately on a glass slide for microfilaria under low $(40 \times)$ magnification by a trained $\mathrm{MOH}$ microscopist. The microfilariae were not counted; results were expressed for each individual as 'positive' or 'negative'. Laboratory results were recorded on the original (field) registration form. Microfilaria prevalence was expressed as a percent (number positive divided by number examined $\times 100)$, and classified as hypoendemic (microfilaridermia rate $\leq 40 \%$ ), mesoendemic (microfilaridermia rate $\geq 40-59 \%$ ) or hyperendemic (microfilaridermia $\geq 60 \%$ ) (WHO Report 1991). 
M. Katabarwa et al. Onchocerciasis transmission in Cameroon and Uganda

Table 2 Cameroon (West Province) and Uganda. Baseline and follow-up data on microfilaria and nodules in children under 10 years of age

\begin{tabular}{|c|c|c|c|c|c|c|c|c|c|c|c|}
\hline \multirow[b]{2}{*}{ Country } & \multirow[b]{2}{*}{$\begin{array}{l}\text { District } \\
\text { (No. of communities) }\end{array}$} & \multicolumn{5}{|c|}{ Baseline } & \multicolumn{5}{|c|}{ Follow-up } \\
\hline & & Year & $\begin{array}{l}\text { No. } \\
\text { assessed }\end{array}$ & $\begin{array}{l}\text { Mean } \\
\text { age }\end{array}$ & $\% \mathrm{mf}$ & $\begin{array}{l}\% \\
\text { Nodules }\end{array}$ & Year & $\begin{array}{l}\text { No. } \\
\text { assessed }\end{array}$ & $\begin{array}{l}\text { Mean } \\
\text { age }\end{array}$ & $\% \mathrm{mf}$ & $\begin{array}{l}\% \\
\text { Nodules }\end{array}$ \\
\hline \multirow{5}{*}{$\begin{array}{l}\text { Cameroon } \\
\text { (West Province) }\end{array}$} & Bangangte (Community) (2) & 1996 & $\mathrm{~N} / \mathrm{A}$ & $\mathrm{N} / \mathrm{A}$ & $\mathrm{N} / \mathrm{A}$ & $\mathrm{N} / \mathrm{A}$ & 10 & 87 & 6.6 & 3.4 & 0 \\
\hline & Foumbot (2) & 1996 & 83 & 5.64 & 50.60 & 51.81 & 10 & 50 & 3.7 & $6^{*}$ & $0 *$ \\
\hline & Bafang (2) & 1996 & 72 & 5.7 & 6.94 & 0 & 10 & 77 & 5.5 & $0 * *$ & 0 \\
\hline & Kekem (2) & 1996 & 30 & 6 & 26.67 & 0 & 10 & 98 & 6.1 & $0 *$ & 0 \\
\hline & Bandja (2) & 1996 & $\mathrm{~N} / \mathrm{A}$ & $\mathrm{N} / \mathrm{A}$ & $\mathrm{N} / \mathrm{A}$ & $\mathrm{N} / \mathrm{A}$ & 10 & 136 & 7.1 & 8.1 & 0 \\
\hline Subtotal & 10 & & 185 & 5.78 & 29.73 & 23.24 & & 448 & 5.80 & $3.8 *$ & $0^{*}$ \\
\hline \multirow[t]{5}{*}{ Uganda } & Kasese (3) & 1993 & 25 & $\mathrm{~N} / \mathrm{A}$ & 8.00 & 0 & 13 & 10 & $\mathrm{~N} / \mathrm{A}$ & 0 & 0 \\
\hline & Kisoro (5) & 1993 & 32 & $\mathrm{~N} / \mathrm{A}$ & 15.60 & 0 & 13 & 9 & $\mathrm{~N} / \mathrm{A}$ & 0 & 0 \\
\hline & Kanungu (2) & 1993 & 32 & $\mathrm{~N} / \mathrm{A}$ & 3.13 & 0 & 13 & 16 & $\mathrm{~N} / \mathrm{A}$ & 0 & 0 \\
\hline & Moyo (4) & 1993 & 32 & $\mathrm{~N} / \mathrm{A}$ & 40.60 & 0 & 13 & 54 & $\mathrm{~N} / \mathrm{A}$ & $1.9^{*}$ & 0 \\
\hline & Nebbi (4) & 1993 & 56 & $\mathrm{~N} / \mathrm{A}$ & 69.60 & 0 & 13 & 41 & $\mathrm{~N} / \mathrm{A}$ & $7.3^{*}$ & 0 \\
\hline Subtotal & 20 & & 177 & $\mathrm{~N} / \mathrm{A}$ & 33.89 & 0 & & 130 & $\mathrm{~N} / \mathrm{A}$ & $3.1 *$ & 0 \\
\hline Grand total & 30 & & 362 & & 31.77 & 11.88 & & 578 & & $3.63 *$ & 0 \\
\hline
\end{tabular}

N/A, not available.

*Significance level $(P<0.0001)$ - follow-up compared with the baseline.

$*$ Significance level $(P<0.05)$ - follow-up compared with the baseline.

\section{Histology of excised nodules}

In sentinel communities of four districts in Uganda (Kasese, Mbale, Moyo and Nebbi districts) nodulectomy was offered to a sample of willing adults. The procedures were performed by trained $\mathrm{MOH}$ clinicians using sterile technique as described by Albiez et al. (1988). Excised nodules were preserved in $90 \%$ ethanol and transported to the Bernhard Nocht Institute for Tropical Medicine in Hamburg, Germany where they were sectioned, stained by H\&E and read by an expert (Prof. D.W. Büttner) following criteria for vitality and fertility of female and male O. volvulus (Duke et al. 2002). The results were compared with findings from an unpublished study of 28 nodules obtained in 1993 and read by the same expert (unpublished results courtesy of Vector Control Division, MOH, Uganda and Prof. D.W. Büttner, Bernhard Nocht Institute).

\section{Treatment coverage}

At the launching of the treatment programme, CDDs in all communities onchocerciasis selected for mass treatment with ivermectin conducted a complete census and results were recorded in a community register. Registers were updated every year by the CDDs, before another round of ivermectin distribution was implemented. We determined treatment coverage of the sentinel communities based on the annual summary treatment statistics kept by the local $\mathrm{MOH}$ offices and The Carter Center country office. Treatment coverage was defined as number of persons treated divided by the eligible population denominator (which is the total population minus children under 5 years of age) determined each year during the treatment exercise. 'Interval treatment coverage' was defined as the average of coverage over the interval (10 years in Cameroon, 13 years in Uganda) for each sentinel village.

\section{Data analysis}

Programme baseline and follow-up nodule and microfilaria prevalence figures for 'Older children and Adults' and 'Young Children' were compared implementing general linear contrasts using Sudaan statistical software version 9.0 (RTI International in North Carolina). Sudaan provides estimates that take into consideration the clustering effect of the districts comprising the sample. Indicators at the community level were compared using the chi-square test for independence (Epi Info Version 6.04; CDC, Atlanta, GA, USA).

\section{Results}

Prevalence changes in older children and adults

Survey results were available for 5269 persons over the age of 10 years. In Cameroon, the baseline survey examined 719 persons and the follow-up survey 838 persons; in Uganda the baseline involved 1590 persons, and 2122 in the follow-up survey (Table 1). All sentinel areas began as 
M. Katabarwa et al. Onchocerciasis transmission in Cameroon and Uganda

hyperendemic for either or both nodule and microfilaria prevalence, with the exception of Kisoro, which was mesoendemic. All sentinel areas showed a significant reduction in the proportion of persons positive for microfilaria $(P<0.0001)$, and nodules $(P<0.0001)$ when baseline and follow-up surveys were compared. In Cameroon, the overall microfilaria prevalence decreased from a hyperendemic mean of $70.1 \%$ [95\% confidence interval $(\mathrm{CI})=57.6-82.6]$ at baseline to a hypoendemic range of $7.04 \%(\mathrm{CI}=0.4-13.0)$. Nodule prevalence decreased from a hyperendemic mean of $58 \%(\mathrm{CI}=42.8-73.2)$ to a hypoendemic mean of $9.55 \%(\mathrm{CI}=6.2-12.9)$. Similarly, in Uganda, microfilaria prevalence among older children and adults decreased from a hyperendemic mean of $71.9 \%$ $(95 \% \mathrm{CI}=44.1-84.7)$ to a hypoendemic mean of $7.5 \%$ (CI $=0-15.8)$ and nodule prevalence from hyperendemic mean of $53.2 \%(\mathrm{CI}=33.8-72.6)$ to a hypoendemic mean of $9.7 \%(\mathrm{CI}=2.3-17.1)$.

In 2005, the highest prevalences among older children/adults were observed in Cameroon's Foumbot District (microfilaria $22.64 \%$ and nodules $16.04 \%$ ) and in Uganda's Nebbi District (microfilaria $19.15 \%$ and nodules $19.52 \%$ ). Foumbot's microfilaria prevalence was significantly higher than in other Cameroonian districts at follow-up, although this had not been so at baseline. In contrast, Nebbi had significantly higher microfilaria and nodule rates than other Ugandan sentinel districts at both baseline and follow-up $(P<0.0001)$.

\section{Prevalence changes in young children}

Survey results were available for 940 children under the age of 10 years. In Cameroon, the baseline survey examined 185 children and the follow-up survey 448 (there were no baseline results from Bangangte and Bandja districts). In Uganda, the baseline involved 177, the follow-up survey 130 (Table 2). Baseline microfilaria prevalences in young children varied widely, ranging from $3.1 \%$ to $69.6 \%$. In contrast, only children $(51.8 \%$ of 42$)$ in Foumbot district were positive for nodules in the baseline survey. Follow-up surveys in Cameroon revealed a drop in microfilaria prevalence among children less than 10 years of age from $29.7 \%(\mathrm{CI}=0.0-66.1)$ to $3.8 \%(\mathrm{CI}=0-4.9)$. In Uganda, microfilaria prevalence of $31.8 \%(\mathrm{CI}=1.5-66.3)$ also decreased in the follow-up survey to $3.6 \%(\mathrm{CI}=0.0-6.7)$. Confidence intervals were wide given the baseline variance of prevalences, but chi-square testing for change was highly significant $(P<0.0001)$. Positive children were found in Bangangte $(3.4 \%)$, Foumbot $(6 \%)$ and Banja $(8.1 \%)$ in Cameroon, and in Moyo (1.9\%) and Nebbi $(7.3 \%)$ in Uganda. Nodule prevalence was zero in all follow-up surveys from West Province.

\section{Nodule histological changes}

Histological results of 80 excised nodules from Uganda (Kasese, Mbale, Moyo and Nebbi districts) obtained in 2005 showed that majority of female worms (64\%) and male worms $(81.4 \%)$ were alive, with $24 \%$ of live female worms being inseminated. However, compared (Figure 1) with nodules obtained in 1993, 2005 findings showed a significant reduction $(P<0.05)$ in: $(1)$ live female worms (from $89 \%$ to $63.8 \%$ ); (2) females with intact embryos from $59 \%$ to $11.7 \%$; (3) inseminated female worms (from $56 \%$ to $24.1 \%$ ) and (4) nodules with intact microfilariae (from $64 \%$ to $7 \%$ ). A significant and positive increase of females with oocytes only (from $13 \%$ to $50.4 \%$ ) was also observed. There was no significant change in percentage of live male worms and live male worms with sperm.

\section{Interval treatment coverage}

The sentinel communities in Cameroon achieved a mean treatment coverage of the eligible population of $94 \%$ (range of $37-100 \%$ ) from 1996 to 2005 . In Uganda, sentinel communities achieved a mean coverage of $91.8 \%$ (range of 74-100\%) from 1993 to 2005 (Table 1). Coverage in sentinel communities was not different from other communities under mass treatment in the sentinel and other affected districts in both countries which maintained annual mean treatment coverage of the eligible population of at least $85 \%$ (data not shown). The lower coverages were restricted to the early years of the programme when ivermectin distribution programmes were first established. The information from registers shows a mean annual treatment coverage of eligible population of at least $85 \%$, with the exception of Kekem in Cameroon $(76.7 \%)$. The highest 2005 prevalences observed in Cameroon's Foumbot district and Uganda's Nebbi district had mean coverages of $98.3 \%$ (range 85.7-100) and 93.0\% (range 92-100), respectively.

\section{Discussion}

A decade or more of ivermectin mass treatment has dramatically reduced microfilaria and nodule prevalence in sentinel villages in West Province Cameroon and five districts in Uganda. While the APOCs goal is 'to eliminate onchocerciasis as a disease of public health and socioeconomic importance throughout Africa' (Amazigo \& Boatin 2006), in terms of impact this goal is not clearly defined. Since the APOC partnership supports CDTI implementation only when there is meso- and hyperendemic onchocerciasis, we would propose that areas below those threshold indices (e.g. 'hypoendemic') as having disease levels that are not a public health problem. Using this 
M. Katabarwa et al. Onchocerciasis transmission in Cameroon and Uganda

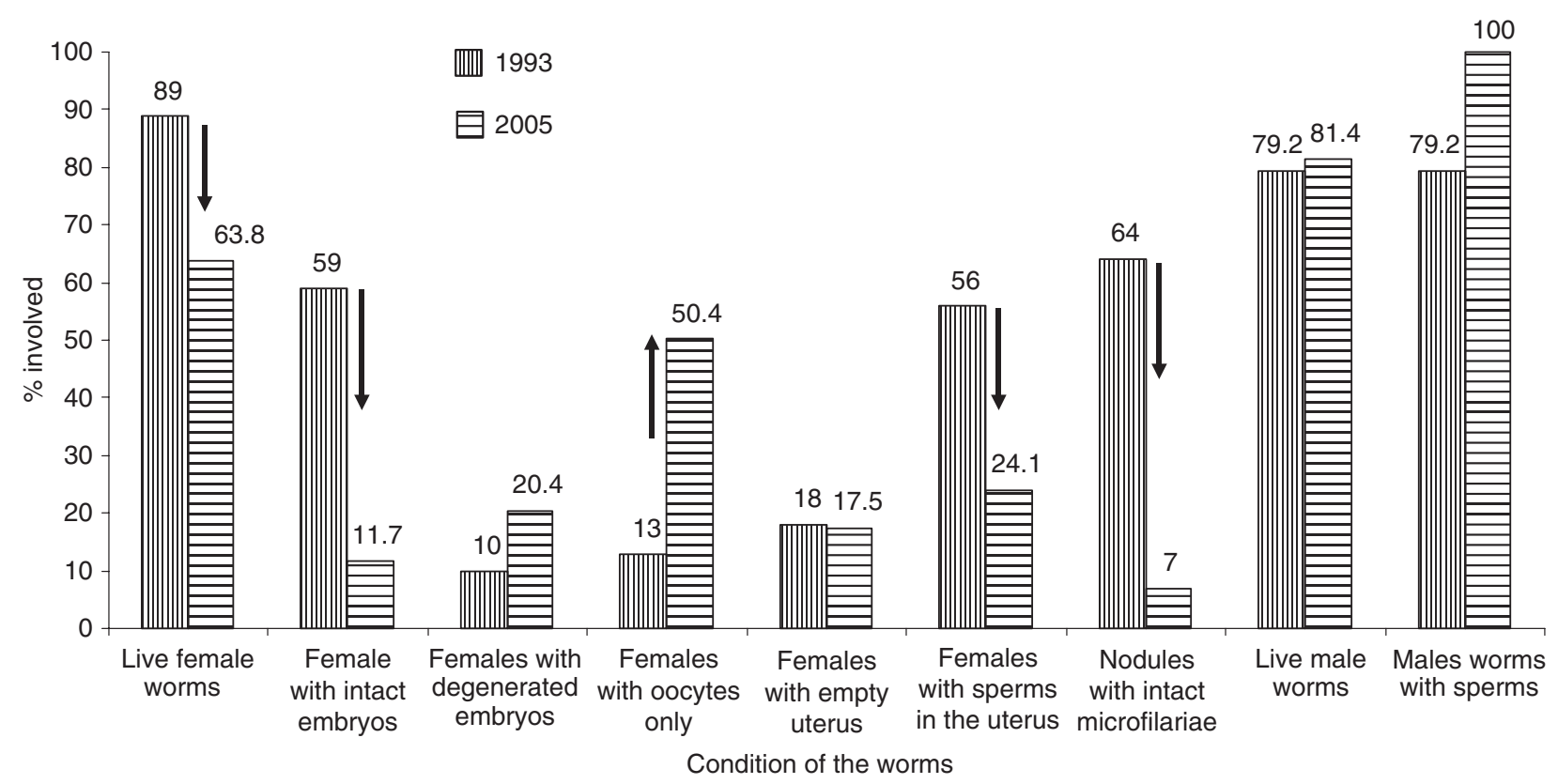

Figure I Uganda: Comparative results of worms in excised onchocercal nodules (1993 and 2005). Histological results of 28 excised onchocercal nodules in a baseline study of 1993 (unpublished results courtesy of Vector Control Division, Ministry of Health (MOH), Uganda and Prof. D.W. Büttner from the Bernhard Nocht Institute for Tropical Medicine in Hamburg) and 80 excised nodules in a followup study of 2005 from Kasese, Mbale, Moyo and Nebbi districts. Arrows show significant changes $(P<0.05)$. Worms observed in 2005 were alive but less reproductively active.

definition, in all cases the APOC goal has been achieved. We note, however, that the $19.5 \%$ nodule prevalence in Nebbi district in Uganda and the $16.0 \%$ nodule prevalence in Foumbot district in Cameroon remain uncomfortably close to the proposed threshold, even after over a decade of ivermectin treatment. This threshold is controversial, and we note that others suggest that intensity of microfilaria counts be used to judge thresholds of the 'public health problem', defining a geometric mean of more than 5 microfilaria/mg skin (or per snip) be used to define the limits of 'onchocerciasis as a public health problem' (WHO Report 1991; Remme 2004). We could not use this definition in our study because neither baseline nor 2005 surveys quantified microfilaria counts, and the method used to obtain skin snips could have resulted in a range of skin weights examined.

Onchocerciasis prevalence may have been lowered to a point of no longer being a public health problem, but onchocerciasis transmission appears to continue in many areas. We also found that children under 10 years of age in the follow-up surveys (e.g. born after commencement of annual treatment campaign) had positive skin snips for microfilaria, albeit at much lower rates than before the campaign. There were positive children in $50 \%$ of sentinel areas: three of five in Cameroon and two of five in Uganda. This is an indication of ongoing transmission (and the inability of the annual distribution of ivermectin to reliably interrupt transmission). We also noted that 2005 nodule histological results from Uganda showed that there were still live female worms, inseminated female worms and living male worms, confirming that the risk for infecting black flies remains. Our study findings were consistent with other peer review studies that have shown that an annual dose of ivermectin may not eliminate transmission of onchocerciasis (Remme et al. 1990; Boatin et al. 1998; Winnen et al. 2002; Borsboom et al. 2003; Mas et al. 2006). In contrast, Ndyomugyenyi et al. (2004), in a study comparing the impact of ivermectin treatment alone and in parallel with vector elimination in two parishes of Kamwenge and Kyenjojo districts of Uganda, found that after 11 years of ivermectin distribution alone, children under 10 years in the follow-up survey were negative for microfilaria. The authors note however that the high impact of ivermectin as the only intervention could have been due to ongoing environmental degradation in the study area that resulted in the disappearances of the vector.

Should CDTI mass treatment activities cease in these controlled, but formerly meso- and hyperendemic areas, we believe there is risk of disease recrudescence. This is further complicated by the fact that mass treatment mostly excludes hypoendemic communities and it is possible that infected 
M. Katabarwa et al. Onchocerciasis transmission in Cameroon and Uganda

persons from these untreated areas could 'reseed' the parasite into former meso- and hyperendemic treatment zones, where environmental and vector conditions could consequently contribute to rapid disease recrudescence in the post-treatment scenario.

As APOC financial support comes to an end in 2015, the responsibility of funding and sustaining ivermectin distribution increasingly falls on the governments and $\mathrm{MOH}$ of the endemic countries, along with (in many cases) their non-governmental organizations (NGO) partners. There is evidence that national governments have not provided regular and adequate funding for CDTI projects (Hopkins et al. 2005). Yet, our findings suggest that either annual treatments must continue to be provided, or a new strategy aimed at complete elimination be devised if recrudescence of onchocerciasis is to be avoided in the future.

Long-term mass drug administration poses the risk of emergence of resistance. In a recent report from Ghana, it was suggested that in some treatment areas adult female O. volvulus worms are resuming microfilaria reproduction more rapidly after ivermectin treatment than would normally be expected (Osei-Atweneboana et al. 2007). The data we present show that a decade of annual ivermectin treatment has done extremely well in eliminating onchocerciasis as a public health problem in all areas examined. However, the microfilaria prevalences in Foumbot and Nebbi in the follow-up survey 10 months after the previous ivermectin dose were significantly higher than in other sentinel districts in spite of reported high treatment coverage. This could be explained by a host of reasons other than resistance: First, baseline microfilaria and nodule rates were highest in Foumbot and Nebbi compared with other sentinel areas (Table 1), but significantly higher at baseline only in Nebbi $(P<0.0001)$. Baseline infection rates in young children (reflecting the force of infection) were also highest in Foumbot and Nebbi districts. It is likely that higher vector density in these areas (related to the force of infection) were able to maintain transmission here (Borsboom et al. 2003). The second, but less likely explanation could be that reported treatment coverage in these areas may not be valid. Reliability of treatment coverage reports was not assessed; however, we have no reason to believe that treatment coverage reports from Foumbot and Nebbi were less reliable than those from other areas in this study. Lastly, although $\mathrm{mf}(\mathrm{mf}$ prevalence is still the best method of diagnosing onchocerciasis. As mf prevalence reduces below where detection becomes difficult, other methods such as nodule prevalence may be commonly used. However, subcuteneous swellings from other aetiologies could be confused for onchocerciaisis resulting into overdiagnosis of onchocerciasis) prevalence rates from skin snips method are valid findings, misdiagnosis of nodules (other aetiologies for subcutaneous swellings with the clinical characteristics of onchocercal nodules) becomes of great probability as onchocerciasis prevalence decreases (Boatin et al. 2002; Katabarwa et al. 2008). Our observations in Foumbot and Nebbi districts require further field study and close follow-up.

The results of the present paper indeed build upon other peer review publications that annual distribution of ivermectin will not stop transmission of onchocerciasis in meso- and hyperendemic areas. Our findings extend these observations to periods of 13 years. Based on this study, we recommend that annual ivermectin distribution not be discontinued after 15 years, and that governments and communities are clearly informed that they should plan and support ivermectin distribution indefinitely. We also recommend the full implementation of the recommendations of the 2001 Conference on Eradicability of Onchocerciasis for the investigation of new ways of applying current tools to completely interrupt transmission, and the development of better diagnostic tools and more effective treatments and macrofilaricides (Dadzie et al. 2003).

\section{Conclusion}

Results from over 6200 examinations over a decade or more (10 years in Cameroon, and 13 years in Uganda) show that an annual dose ivermectin treatment has reduced meso- and hyperendemic onchocerciasis to hypoendemic prevalences (a community nodule rate of $\leq 20 \%$ and a microfilaria prevalence of $\leq 40 \%$ ), which we consider as having achieved the goal of elimination of onchocerciasis as a 'public health problem'. However, over $3 \%$ of children under 10 years of age examined in 2005 had microfilaria in skin, and excised nodules still showed live and inseminated female worms, implying that onchocerciasis transmission persists. Until new strategies are implemented that can halt transmission of O. volvulus, annual dose of ivermectin treatment should be continued indefinitely in formerly meso- and hyperendemic onchocerciasis zones.

\section{Acknowledgements}

The authors would like to acknowledge the Uganda 2005 onchocerciasis surveillance team members: Mr E. Tukesiga, J. Katamanywa, J. Wamani, E. Banoba, N. Ogweng and L. Hakiri. Dr Oboth Okoth, Medical officer (MO), Kanungu, and Dr Chandia (MO), Moyo district surgically removed nodules from patients in 2005. In Cameroon, we acknowledge Ms Catherine Mentou, the Programme Officer, Carter Center, Cameroon office, and the district health personnel: Dr Mfokue Bouba, Medical Officer, Foumbot health district; Dr Allassa Mounton, Medical Officer, 
M. Katabarwa et al. Onchocerciasis transmission in Cameroon and Uganda

Bangangté health district; Dr Joseph Pouenpène, Medical Officer, Bafang health district; and Dr Abel Fossi, Medical Officer, Bandja health district; Mr Maurice Ngoungoué, Health Bureau chief; and programme coordinators, $\mathrm{Mr}$ Leonard Tchana and Mr Demanga Ngangue who participated in the 2005 surveys.

We would also like to acknowledge Prof. D.W. Büttner from the Bernhard Nocht Institute for Tropical Medicine in Hamburg, Germany who analyzed all the excised nodules from Uganda and Mrs Jacqueline Roberts of Centers for Disease Control and Prevention (Atlanta) who assisted with statistical analysis using Sudaan statistical software, version 9.0. Financial support: The Carter Center, Lions Clubs International (SightFirst Programme), the River Blindness Foundation (for baseline surveys) and the APOC. Ivermectin (Mectizan $\left.{ }^{\circledR}\right)$ is donated by Merck \& Co.

\section{References}

Albiez EJ, Büttner DW \& Duke BO (1988) Diagnosis and extirpation of nodules in human onchocerciasis. Tropical Medicine and Parasitology 39(Suppl. 4), 331-346.

Amazigo U \& Boatin B (2006) The future of onchocerciasis control in Africa. Lancet 368, 1947.

Amazigo UV, Brieger WR, Katabarwa M et al. (2002) The challenges of community-directed treatment with ivermectin (CDTI) within the African Programme for Onchocerciasis Control (APOC). Annals of Tropical Medicine and Parasitology 96, S75-S92.

Boatin BA, Hougard JM, Alley ES et al. (1998) The impact of Mectizan on the transmission of onchocerciasis. Annals of Tropical Medicine and Parasitology 92, S46-60.

Boatin BA, Toe L, Alley ES et al. (2002) Detection of Onchocerca volvulus infection in low prevalence areas: a comparison of three diagnostic methods. Parasitology 125, 545-552.

Borsboom GJJM, Boatin AB, Nagelkerke NJD et al. (2003) Impact of ivermectin on onchocerciasis transmission: assessing the empirical evidence that repeated ivermectin mass treatment may lead to elimination/eradication in West Africa. Filaria Journal 2, 8.

Chippaux JP, Boussinesq M \& Prod'hon J (1995) The use of ivermectin in the control of onchocerciasis. Sante 5, 149-158.

Dadzie Y, Neira M \& Hopkins D (2003) Final report of the conference on the eradicability of Onchocerciasis. Filaria Journal 2, 2.

Duke BO, Marty AM, Peet DL et al. (2002) Neoplastic change in Onchocerca volvulus and its relation to ivermectin treatment. Parasitology 125, 431-444.

Hopkins DR, Richards FO \& Katabarwa M (2005) Whither onchocerciasis control in Africa. American Journal of Tropical Medicine and Hygiene 72, 1-2.

Katabarwa M, Onapa AW \& Nakileza B (1999) Rapid epidemiological mapping of onchocerciasis in areas of Uganda where
Simulium neavei sl is the vector. East African Medical Journal 76, 440-446.

Katabarwa MN, Habomugisha P \& Agunyo S (2002) Involvement and performance of women in community-directed treatment with ivermectin for onchocerciasis control in Rukungiri District, Uganda. Health and Social Care in the Comminity 10, 382-393.

Katabarwa M, Lakwo T, Habumogisha P et al. (2008) Could neurocysticercosis be the cause of onchocerciasis-associated' epileptic seizures? American Journal of Tropical Medicine and Hygiene 78, 400-401.

Mas J, Ascaso C, Escaramis G et al. (2006) Reduction in the prevalence and intensity of infection in Onchocerca volvulus microfilariae according to ethnicity and community after 8 years of ivermectin treatment on the island of Bioko, Equatorial Guinea. Tropical Medicine and International Health 11, 1082-1091.

Molyneux DH \& Davies JB (1997) Onchocerciasis control: moving towards the millennium. Parasitology Today 13, 418-425.

Ndyomugyenyi R, Tukesiga E, Buttner DW et al. (2004) The impact of ivermectin treatment alone and when in parallel with Simulium neavei elimination on of onchocerciasis in Uganda. Journal of Tropical Medicine and International Health 9, 882-886.

Ngoumou P, Walsh JF \& Mace JM (1994) A rapid mapping technique for the prevalence and distribution of onchocerciasis: a Cameroon case study. Annals of Tropical Medicine and Parasitology 88, 463-474.

Osei-Atweneboana MY, Eng JK, Boakye DA et al. (2007) Prevalence and intensity of Onchocerca volvulus infection and efficacy of ivermectin in endemic communities in Ghana: a two-phase epidemiological study. Lancet 16, 2021-2029.

Prost A \& Prod'hon J (1978) Le diagnostique parasitologique de l'onchocercose. Revue critique des méthods en usage. Médicine Tropicale 38, 519-532.

Remme JHF (2004) Research for control: the onchocerciasis experience. Tropical Medicine and International Health 9, 243-254.

Remme J, De Sole G, Dadzie KY et al. (1990) Large scale ivermectin distribution and its epidemiological consequences. Acta Leidensia 59, 177-191.

Richards F, Hopkins D \& Cupp E (2000) Programmatic goals and approaches to onchocerciasis. Lancet 355, 1663-1664.

Rodriguez-Perez MA \& Reyes-Villanueva F (1994) The effect of ivermectin on the transmission of Onchocerca volvulus in southern Mexico. Salud Publica de Mexico, Instituto Nacional de Salud Publica 36, 281-290.

Taylor HR, Pacque M, Munoz B et al. (1990) Impact of mass treatment of onchocerciasis with ivermectin on the transmission of infection. Science 250, 116-118.

WHO Report (1991) Strategies for Ivermectin Distribution Through Primary Health Care System. (WHO/PHL/91.24) WHO, Geneva.

Winnen M, Plaisier AP, Alley ES et al. (2002) Can ivermectin mass treatments eliminate onchocerciasis in Africa? Bulletin of World Health Organization 80, 384-391.

Corresponding Author Moses Nayenda Katabarwa, Program Epidemiologist, Emory University, The Carter Center, 1149 Ponce de leon, Atlanta, GA 30306, USA. Tel.: +1 770488 4511; Fax: +1 770488 4521; E-mail: mkataba@emory.edu 